\title{
Confocal laser scanning microscopy, optical coherence tomography and transonychial water loss for in vivo investigation of nails
}

\author{
E. Sattler, R. Kaestle, ${ }^{\star}$ G. Rothmund* and J. Welzel* \\ Department of Dermatology and Allergology, Ludwig-Maximilians-University of Munich, Germany \\ *Department of Dermatology and Allergology, General Hospital Augsburg, Sauerbruchstraße 6, 86179 Augsburg, Germany
}

\section{Summary}

\author{
Correspondence \\ Julia Welzel. \\ E-mail: julia.welzel@klinikum-augsburg.de
}

Funding sources

The Gesellschaft zur Förderung des

Zentralklinikums Augsburg e. V., Augsburg,

Germany helped in funding the confocal

microscopy device.

\section{Conflicts of interest}

R.K. and J.W. have a cooperation agreement regarding scientific evaluation of the method and training courses for confocal microscopy with Mavig (provider of the confocal laser scanning microscopy device, Vivascope 1500). E.S. and G.R. have none to declare.
Background Nail disorders can be diagnostically challenging to the dermatologist. Noninvasive methods might help to avoid nail biopsies. More knowledge of the typical features of healthy nails with these techniques is needed for comparison with nail diseases. Objectives To describe the typical morphology of healthy nails in optical coherence tomography (OCT) and confocal laser scanning microscopy (CLSM) and to examine the influence of exposure to water on OCT features, nail thickness as well as on transonychial water loss (TOWL) before and after a hand bath.

Material and methods In the first part healthy nail plates were investigated with OCT and CLSM. The thickness of layers as well as structural details were defined. Secondly, in a prospective study 30 healthy volunteers conducted 10 hand baths with water within a 2 -week period. Measurements of nail thickness and signal intensity by OCT as well as of the TOWL were performed over time.

Results In OCT the unaffected nail plate appears as a band-like, layered structure, yet with some individual differences. In addition, CLSM is able to display single corneocytes and the integrity of their borders. Exposure to water led to a transient rise of TOWL and in nail thickness.

Conclusions OCT, CLSM and TOWL offer valuable noninvasive diagnostic tools for the examination of nails. Using CLSM, microscopic features like integrity of single corneocytes can be investigated. OCT allows determination of nail plate thickness and both OCT and TOWL measurements are able to detect short-term effects after exposure to water.
Nail diseases are often very troublesome to the patient and can be diagnostically challenging to the dermatologist. To reduce the number of nail biopsies, noninvasive technologies are of high interest in the diagnosis of nail disorders. Optical coherence tomography (OCT) and confocal laser scanning microscopy (CLSM) offer a quick and noninvasive view of the patients' nails in real time. While few data are available on OCT and nails, no reports exist so far on CLSM and healthy nails. To be able to assess changes in affected nails, knowledge is needed on the typical morphology of healthy nails in OCT and CLSM and of changes due to exposure to water.

\section{Material and methods}

\section{Morphological studies using confocal laser scanning microscopy and optical coherence tomography}

Morphological studies of healthy nail plates were conducted by CLSM as well as OCT in healthy individuals in a standardized setting. For OCT 33 healthy nail plates of 33 test subjects aged
19-53 years were imaged and evaluated using the OCT device, Callisto (Thorlabs AG, Lübeck, Germany). For CLSM 15 fingernails of 10 healthy individuals were imaged using the reflection mode of the Vivascope 1500 (Mavig, Munich, Germany). The device was placed on the middle of the nail plate analogous to the measurements with OCT. Images were gathered with the 'VivaStack' function going down in horizontal layers of the same area (middle of the nail plate) with steps of 15-20 $\mu \mathrm{m}$ with single images of $500 \times 500 \mu^{2}$. Typical features and thickness of layers as well as individual differences were evaluated. In line with the prospective study on transonychial water loss (TOWL) and on OCT, in addition, CLSM of one middle finger was performed before and after a 30 -min $38{ }^{\circ} \mathrm{C}$ hand bath.

\section{Prospective study on changes in optical coherence tomography features and transonychial water loss after water exposure}

In a prospective experimental study, 30 healthy volunteers were recruited for imaging of the nail by OCT and measure- 
ments of nail thickness as well as of TOWL were performed. After written informed consent the test subjects conducted a daily hand bath $\left(38{ }^{\circ} \mathrm{C}\right.$ for $\left.30 \mathrm{~min}\right)$ of their middle finger every Monday through Friday over two consecutive weeks (total of 10 hand baths). Sides were varied between test subjects. The dominant hand (handedness) was registered.

\section{Measurements}

Measurements (OCT and TOWL) were performed on day 1 (Monday of the first week) before the first hand bath as well as right after the 30-min hand bath, $15 \mathrm{~min}$ later, $30 \mathrm{~min}$ later and $4 \mathrm{~h}$ after completing the exposure to water. On day 12 (Friday of the second week) measurements were performed again at the same time points as on day 1. Measurement of TOWL was conducted with a Tewameter (Courage \& Khazaka, Cologne, Germany). The measurement of the nail thickness and of the optical density was performed with the OCT device, Callisto (Thorlabs AG). Images were taken in the middle of the nail plate lengthwise over $4 \mathrm{~mm}$ with a nominal resolution of $10 \mu \mathrm{m}$ and with a corrected refractive index of $1 \cdot 3$. Underneath the entrance signal peak within the upper part of the nail plate, the coefficient of signal reduction $[\mu]$ was taken as a measure for light scattering.

\section{Analysis of data}

The graphic presentations show the average value of the starting point differences (differences between the current value and the initial value on day 1). Differences between the different time points of measurement were analysed with the original data as well as with the starting point differences with the Wilcoxon pair difference test for linked random samples. Statistical significance was assumed at a P-value $\leq 0.05$.

Next, the area under the curve (AUC) of the starting point differences was calculated separately for each day of measuring, starting from the value right after treatment. For this calculation the starting point differences were referred to the first measured value of that specific day to evaluate only acute effects. Again the Wilcoxon pair difference test was used to check for statistical significance.

\section{Results}

\section{Confocal laser scanning microscopy: morphological features of healthy nails}

Similar to the skin, CLSM of the nail is able to offer detailed information in almost histological resolution. Using the 'VivaStack' function, the nail plate can be scanned from the surface to the transition of the plate to the underlying nail bed in horizontal images. As with OCT (see below), different layers can be differentiated by CLSM according to the intensity of reflection. The superficial layer shows a brighter reflection, followed by a zone with slightly poorer signal, again followed by a brighter zone in the depth. While these layers and their thicknesses are better seen and measured in OCT (see below), the transition to the underlying nail bed is clearly visible and displayed in wave-like structures, which are directed towards the fingertip. The main advantage, though, of using the CLSM technique in healthy nails is the depiction of detailed information on single cells. Within the nail plate single corneocytes can be investigated. Figures 1 and 2 demonstrate VivaStack images from the surface through all zones of the nail plate down to the nail bed. In contrast to the regularity of a normal nail, leukonychia is caused by a detachment of single corneocytes (Fig. 3). A nail with mycotic infection displays in detail even the hyphae of the dermatophytes thereby allowing an in vivo diagnosis of onychomycosis (Fig. 4).

\section{Optical coherence tomography: morphological features of healthy nails}

In OCT, the unaffected nail plate appears as a band-like structure usually with a homogenous intensity of signal in
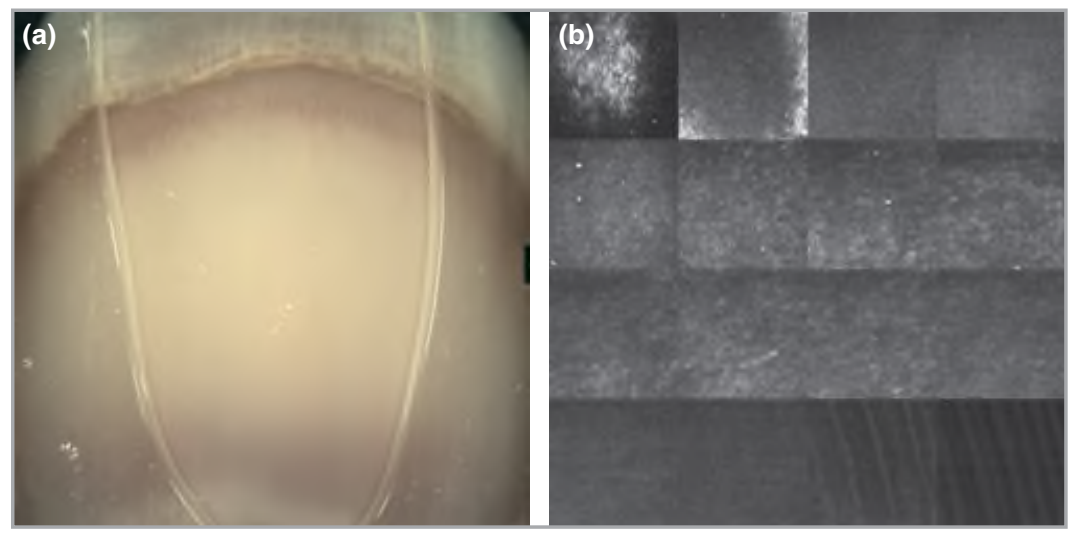

Fig 1. (a) Macroscopic image of the thumbnail plate of the left hand. (b) VivaStack image of the thumbnail of the left hand. Single images $\left(500 \times 500 \mu \mathrm{m}^{2}\right)$ with $30-\mu \mathrm{m}$ downwards steps. Zones of different brightness can be differentiated going deeper into the nail plate, correlating to the layers as described for optical coherence tomography (see Fig. 5). In the depth the transition from the nail plate to the underlying nail bed can be visualized, presenting as wave-like structures, which are directed towards the fingertip. 

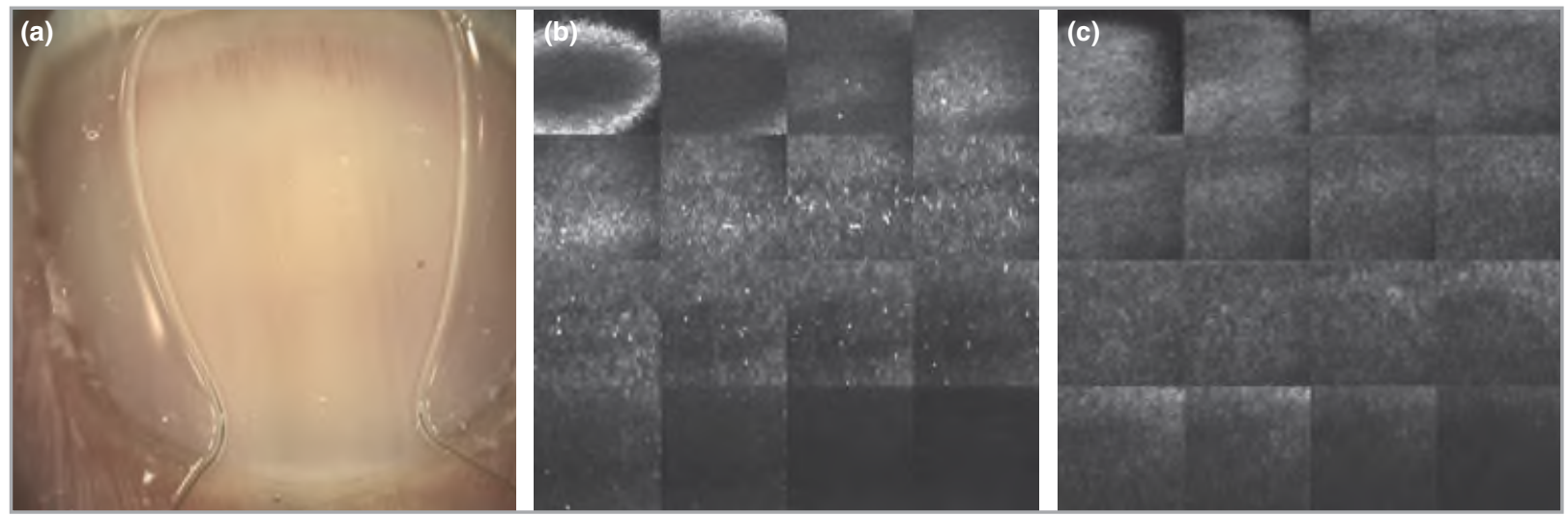

Fig 2. (a) Macroscopic image of the left middle fingernail plate. (b) VivaStack image of the same finger. Single images $\left(500 \times 500 \mu \mathrm{m}^{2}\right)$ with $15-\mu \mathrm{m}$ downwards steps before a hand bath. (c) VivaStack image of the same nail unit. Single images $\left(500 \times 500 \mu \mathrm{m}^{2}\right)$ with $15-\mu \mathrm{m}$ downwards steps right after a 30-min hand bath. Compared with Figure $2 \mathrm{~b}$ the spotty brighter reflections are missing and especially the upper layers appear swollen and blurry as a sign of possible soakage and more hydration.
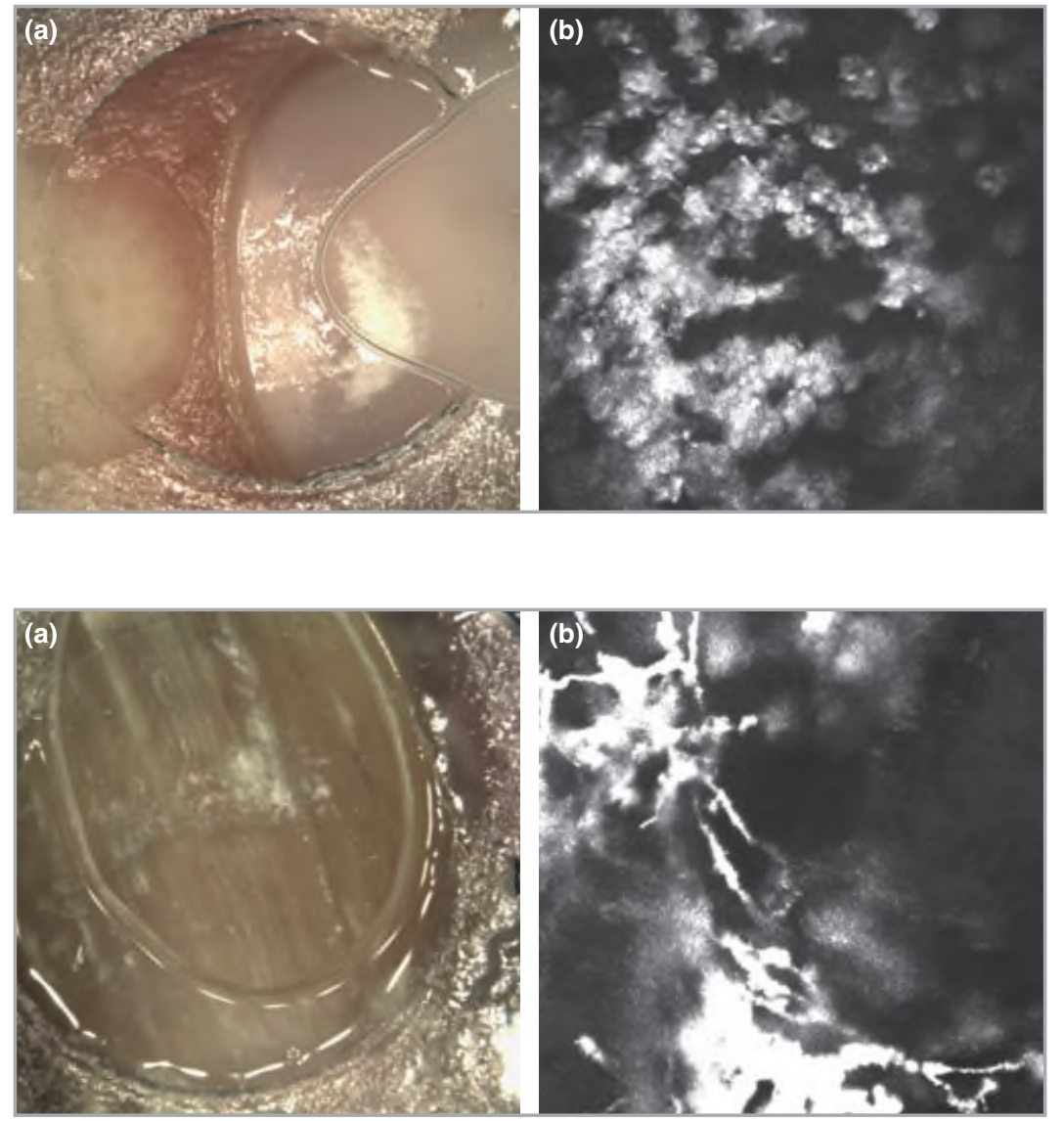

Fig 3. (a) Macroscopic image of the middle finger of the left hand, presenting with a leukonychia in the middle of the nail plate. (b) Confocal laser scanning microscopy image of the same finger at a depth (z) of about $340 \mu \mathrm{m}$. The disturbance of the integrity of the corneocytes can be seen clearly, corresponding to acantholysis seen in histopathological sections.
Fig 4. (a) Macroscopic image of the first toenail, presenting typical clinical features of onychomycosis. (b) Confocal laser scanning microscopy image of the same finger at a depth (z) of about $350 \mu \mathrm{m}$. The hyphae of the dermatophytes can be distinguished easily as lengthy structures with high reflection. the upper layers, more signal-intense layers in the middle and a good demarcation of the underlying nail bed (Fig. 5). Yet some of the nails showed individual differences. The parallel pattern within the nail plate was sometimes very distinct (Fig. 5) but in some test subjects missing almost completely. A few nails displayed further interesting findings such as additional inclined lines (Fig. 6). Other individual features were punctuated spots of high intensity or irregularities in the nail surface leading to signal shadows.

\section{Transonychial water loss and optical coherence tomography features: changes after exposure to water}

Thirty healthy volunteers participated in this prospective experimental study, 24 women and six men aged from 19 to 
Fig 5. Typical layered structure of the healthy nail plate in optical coherence tomography (same nail as imaged by confocal laser scanning microscopy in Figure 1).
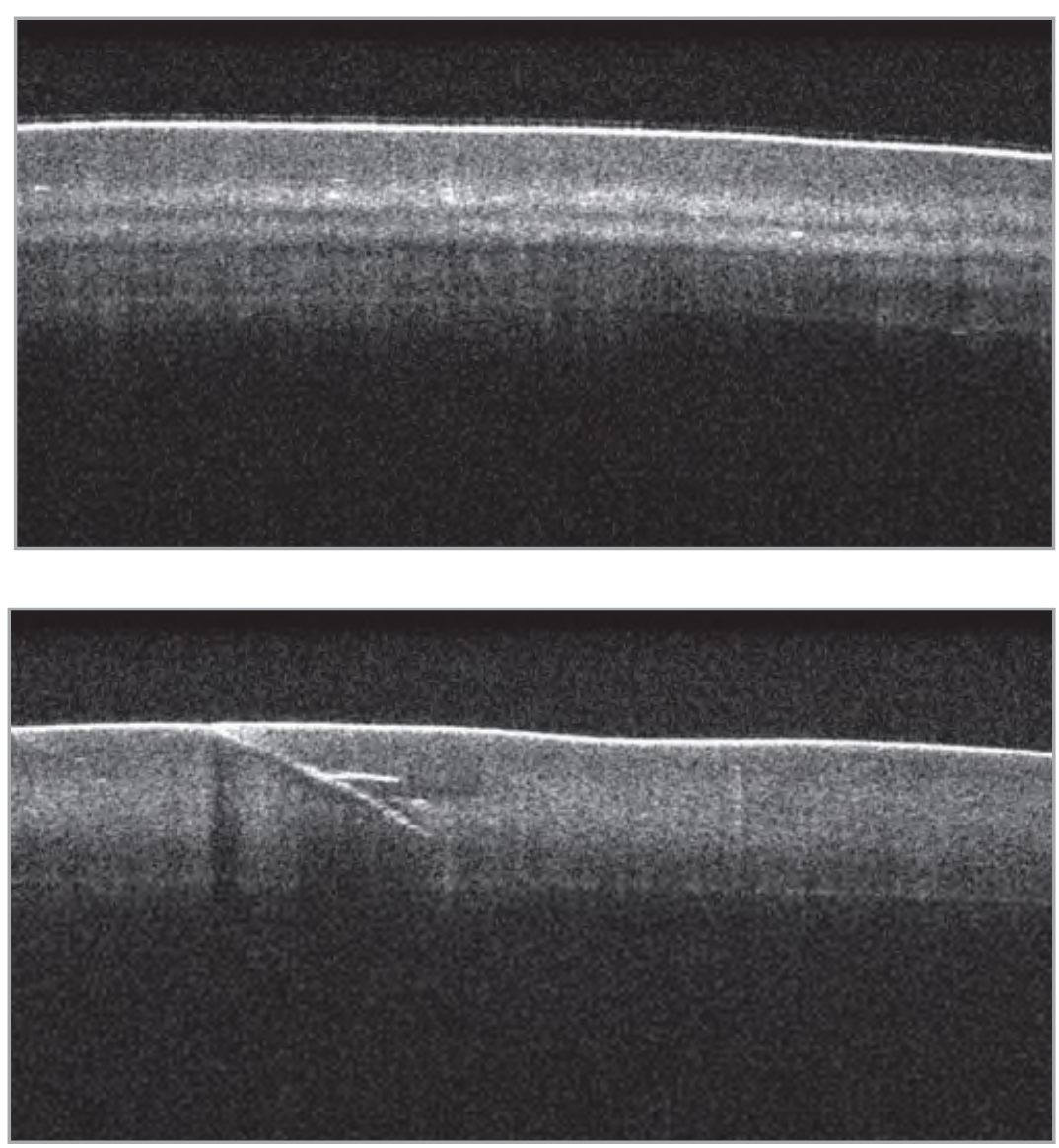

Fig 6. Defect of the nail surface with inclined line into the nail plate.

53 years (median 45 years). Nineteen of the 30 test subjects bathed the left middle finger in water, 11 the right one, designated randomly.

\section{Transonychial water loss}

On both days, the hand bath led to a peak in water evaporation over the nail plate directly after the bath with a maximum value of $101.6 \mathrm{~g} \mathrm{~m}^{-2} \mathrm{~h}^{-1}$. The rise of TOWL reached statistical significance. There were no differences between the two hand baths or between the two initial values on days 1 and 12. The peak was detectable only temporarily for a few minutes after the bath (Fig. 7).

\section{Optical coherence tomography}

The measurements of the nail thickness showed a slight rise after the bath, which reached statistical significance on day 1 and persisted for only a few minutes (short-term effect). There were no differences between the initial values of day 1 and day 12 (Fig. 8). The average nail thickness was $10 \%$ lower in the 24 female test subjects (mean of $453.7 \mu \mathrm{m}$ ) compared with the six male test subjects (mean of $495 \mu \mathrm{m}$; no statistical significance). After correcting for the handedness of the test subjects the nail thickness was significantly higher $(P=0.04)$ on the middle finger of the dominant hand, with the mean thickness for the middle finger of the dominant hand being $465.8 \mu \mathrm{m}$ and for the nondominant hand being $451 \cdot 7 \mu \mathrm{m}$.

\section{Intensity of scattering}

The hand bath led, on days 1 and 12, to a drop of the intensity of scattering in the upper nail plate right after the bath. The drop of the coefficient of light diffusion after water exposure reached significance. Again this was a short-term effect with normalization to initial values after $15 \mathrm{~min}$. No difference was found between the initial values of day 1 and day 12 (Fig. 9).

\section{Morphological changes in optical coherence tomography features before and after the hand bath}

The typical morphological features in OCT underwent mainly three changes due to the exposure to water (Fig. 10):

1 The nail plate appeared thicker right after the hand bath with the layered structures displaying slightly less density.

2 The intensity of scattering directly underneath the signal of accession shows a narrow layer with poorer signal after the hand bath.

3 The demarcation of the underlying nail bed is slightly more diffuse compared with the clear-cut margin from nail to nail bed in the images before the hand bath. 


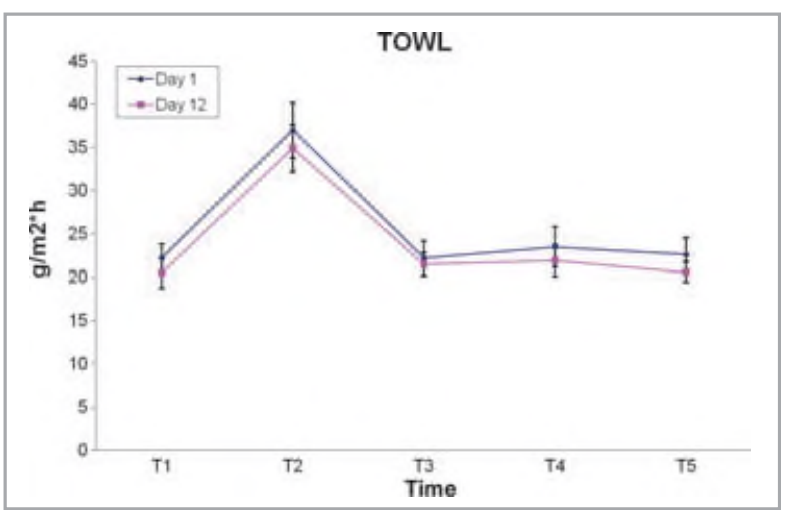

Fig 7. Rise of transonychial water loss (TOWL) directly after the hand bath on day 1 and day 12 over time (T1, before the hand bath; T2, directly after the hand bath; T3, 15 min after the end of the hand bath; T4, 30 min afterwards; T5, $4 \mathrm{~h}$ afterwards). The error bars show the SEM value.

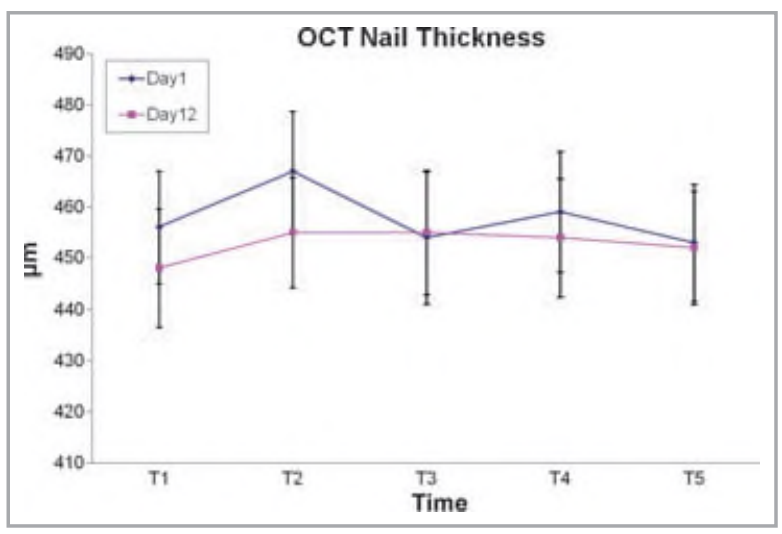

Fig 8. Thickness of the nail plate measured by optical coherence tomography (OCT) dependent on time (T1, before the hand bath; T2, directly after the hand bath; T3, 15 min after the end of the hand bath; T4, 30 min afterwards; T5, $4 \mathrm{~h}$ afterwards). The error bars show the SEM value.

\section{Discussion}

While CLSM is well established as a helpful noninvasive tool with almost histological resolution in the diagnosis of lesions of the human skin, primarily in pigmented lesions but also in other skin tumours and diseases, ${ }^{1-7}$ to our knowledge so far no data are available on the morphological features of CLSM of the healthy nail plate. Only one report is available on CLSM and diseased nails, in which CLSM was able to confirm the diagnosis of onychomycosis in one patient. ${ }^{8}$

The central findings on the morphology of healthy nails in CLSM are as follows:

1 The layered structures typically displayed in OCT can also be found in CLSM, presenting as zones of images with more and less reflective brightness.

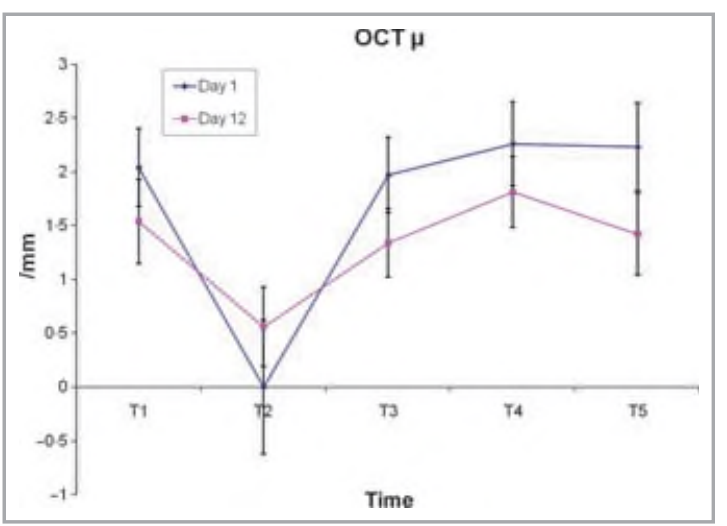

Fig 9. Drop of the coefficient of light diffusion (OCT $\mu$ ) directly after the exposure to water ( $\mathrm{T} 1$, before the hand bath; $\mathrm{T} 2$, directly after the hand bath; T3, 15 min after the end of the hand bath; T4, 30 min afterwards; T5, $4 \mathrm{~h}$ afterwards). The error bars show the SEM value.

2 Compared with the OCT images, which best allow the measurement of thickness of the entire plate and of the different layers, CLSM gives better information on the microscopic structures of the nail plate. Even the borders of the corneocytes can be evaluated and their integrity can be investigated.

3 The value of the knowledge of these features in healthy nails can best be appreciated when compared with diseased nails, especially in disorders causing a problem in normal nail growth, such as onychomycosis, psoriasis or leukonychia (see Figs 3 and 4).

4 There are some limitations. One limitation of CLSM in human skin is the limited depth of accession of about 250$300 \mu \mathrm{m}$. Yet, when imaging healthy nails, depths of even down to about 400-500 $\mu \mathrm{m}$ (see Fig. 2) were reached, probably due to fewer cell organelles absorbing the light on its way forward and backward and a lower refractive index of the nail unit, allowing for gathering more information at greater depth. A second is the limited area of $500 \times 500 \mu \mathrm{m}^{2}$ that can be scanned in a single image. Thirdly, an experienced examiner is needed to be able to capture images with minimal artefacts, as the convex surface of the nail makes positioning and holding of the device during imaging a challenging endeavour. For this problem, a handheld CLSM device such as the Vivascope 3000 might be helpful, as with this device no extra window is needed. Fourthly, an index-matching medium, i.e. oil or ultrasound gel, must be applied onto the surface of the probe before measuring. The whole measurement procedure takes about 5-10 min. Both might be a problem when investigating short-term effects of hydrating agents in contrast to OCT and TOWL, techniques which do not affect the probe in any way.

OCT offers a noninvasive imaging technology based on light reflection. It provides real-time images with a possible penetration depth of about $2 \mathrm{~mm}$ and a resolution of $10 \mu \mathrm{m}$. Similar to transepidermal water loss (TEWL) or TOWL, OCT 


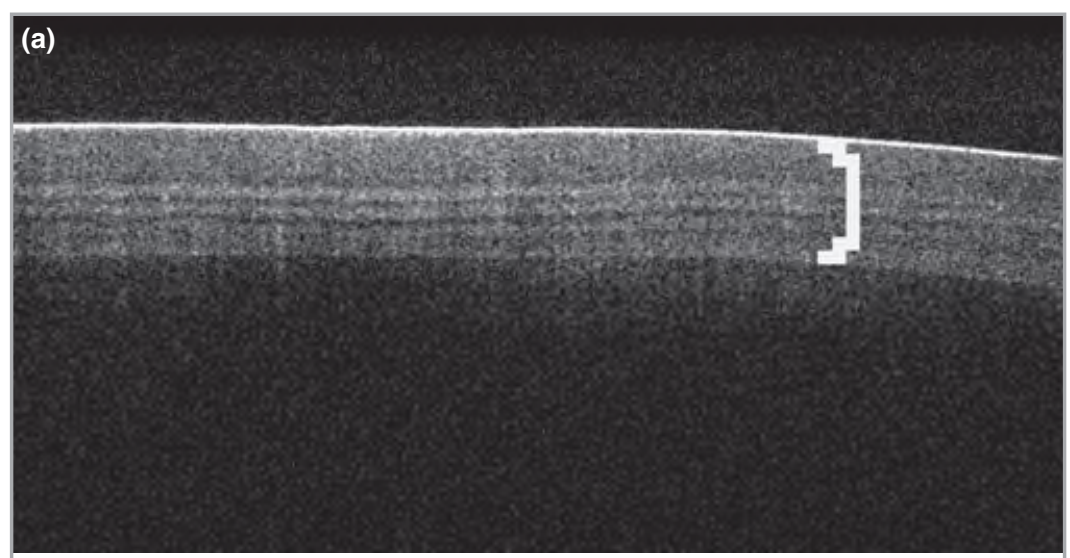

Fig 10. The nail of the left middle finger before (a) and right after the hand bath (b). The nail plate (] in Fig. 10a) appears slightly
thicker after the bath. The intensity of scattering directly underneath the signal of accession $(\leftarrow)$ shows a narrow layer of poorer signal.

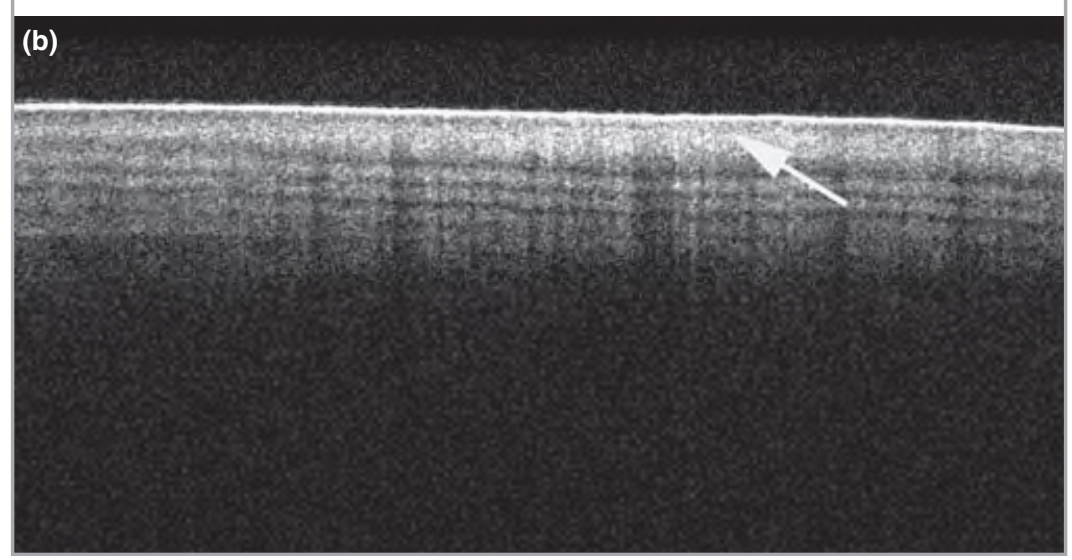

is well established for the examination of the human skin ${ }^{9-11}$ but just evolving for the study of nail disorders.

The typical morphological features we could see using OCT in healthy nails support the preliminary findings of another study with 10 healthy volunteers. This group compared polarization-sensitive OCT, high-frequency $(20 \mathrm{MHz})$ ultrasound and calliper measurements for nail investigation. ${ }^{12}$ Consistent with our data they describe the nail plate as a layered structure containing a varying number of horizontal homogeneous bands of varying intensity and thickness. They also show that the lunula and leukonychia contained distinct horizontal white bands with strong reflection/scattering. These findings are similar to the individual differences we mentioned above concerning punctuated spots with dorsal signal shadow.

Similar to Mogensen et al., ${ }^{12}$ we found that OCT offers a very helpful device for the measurement of nail thickness with comparable values of average thickness in men and women. Another interesting finding was that the nail plate was thicker on the dominant hand as we corrected for handedness, maybe due to more mechanical exposure. The fact that the nail thickness was $10 \%$ higher in the six male test subjects compared with the 24 female test subjects was not statistically significant due to the small number of male test subjects, but would be consistent with differences in skin thickness dependent on sex obtained with ultrasound.

Due to its ubiquity water is a possible skin irritant of special interest. Its irritancy to human skin has been demonstrated by experiments leading to clinical and histopathological inflam- mation and functional damage, as revealed by increased TEWL. This irritancy potential - especially appreciated in occupational dermatology - might be due to several mechanisms such as $\mathrm{pH}$, hardness, osmolarity and temperature. ${ }^{13}$ The aim of this study was to assess the effect of repeated defined exposure to water of the nail plate in terms of morphology (OCT), thickness (OCT) and TOWL.

In contrast to TEWL, which has been used often in scientific research, ${ }^{14,15}$ only few data are available on the TOWL of human nails. ${ }^{16-18}$ This might be due to the problem of fixing the measuring device on the nail plate and creating a closed chamber, which might be difficult depending on the convexity of the nail plate. In this study a special gluing ring provided by Courage \& Khazaka to fix the Tewameter to the nail plate was used.

The baseline TOWL on day 1 and day 12 showed almost no inter-day variability. Also the mean baseline values of $22 \cdot 2$ and $20.5 \mathrm{~g} \mathrm{~m}^{-2} \mathrm{~h}^{-1}$ on day 1 and day 12 , respectively, are consistent with data from a previous study with 21 healthy volunteers where a median TOWL value of $19.4 \mathrm{~g} \mathrm{~m}^{-2} \mathrm{~h}^{-1}$ was found. ${ }^{16}$ Interestingly, the same group found no significant correlation between nail plate thickness (as measured by ultrasound $20 \mathrm{MHz}$ A-scan) and TOWL, ${ }^{16}$ in contrast to a more recent study which found a strong correlation. ${ }^{18}$ Consistent with our results Murdan et al. ${ }^{18}$ also saw a quick rise in TOWL after wetting the nails, even briefly, but with a longer return to control levels. Yet there was no standardized repeated exposure to water examined comparable to our 
study. Measuring at four time points after the bath revealed the effect to be only short term, as return to initial values was already reached after $15 \mathrm{~min}$. In another study a comparison of TOWL in healthy and affected nails with atopic eczema, psoriasis and onychomycosis showed a significantly lower TOWL in the affected nails. As a possible cause, the formation of a stratum granulosum in these diseased nails in contrast to healthy nail plates is discussed. ${ }^{17}$ The aspect of the transient rise in thickness after water exposure features a new interesting finding. It should also be taken into account that the average water content of the fingernail undergoes seasonal changes. ${ }^{19}$

Also, the significant difference in the drop of the intensity of scattering of the nail plate in OCT after water exposure stated above is a new observation. Both are due to a short-term swelling and hyperhydration of the superficial layers of the nail plate, leading to a signal-poor, low-reflectance zone. These findings should be of value for evaluation of the nail plates of healthy and diseased nails in future studies as well as for quantification of environmental effects such as hydration.

\section{What's already known about this topic?}

- So far no data are available on confocal laser scanning microscopy (CLSM) and healthy nails.

- Preliminary studies compared the measurement of nail thickness by optical coherence tomography (OCT), high-frequency ultrasound and measurements of transonychial water loss (TOWL) in healthy and diseased nails.

\section{What does this study add?}

- Using CLSM and OCT, typical features as well as thickness of layers of healthy nails were defined.

- Possible individual differences are shown and the influence of sex and handedness on nail thickness was assessed.

- In a prospective study the standardized exposure of healthy nails to water led to a rise in nail thickness, in TOWL and to a drop of intensity of scattering.

\section{References}

1 Astner S, Ulrich M. [Confocal laser scanning microscopy]. Hautarzt 2010; 61:421-8.
2 Gonzalez S, Gilaberte-Calzada Y. In vivo reflectance-mode confocal microscopy in clinical dermatology and cosmetology. Int $\mathrm{J}$ Cosmet Sci 2008; 30:1-17.

3 Pellacani G, Longo C, Malvehy J et al. In vivo confocal microscopic and histopathologic correlations of dermoscopic features in 202 melanocytic lesions. Arch Dermatol 2008; 144:1597-608.

4 Pellacani G, Scope A, Ferrari B et al. New insights into nevogenesis: in vivo characterization and follow-up of melanocytic nevi by reflectance confocal microscopy. J Am Acad Dermatol 2009; 61:1001-13.

5 Pellacani G, Vinceti M, Bassoli S et al. Reflectance confocal microscopy and features of melanocytic lesions: an internet-based study of the reproducibility of terminology. Arch Dermatol 2009; 145: 1137-43.

6 Ulrich M, Stockfleth E, Roewert-Huber J et al. Noninvasive diagnostic tools for nonmelanoma skin cancer. Br J Dermatol 2007; 157 (Suppl. 2):56-8.

7 Astner S, Gonzalez S, Cuevas J et al. Preliminary evaluation of benign vascular lesions using in vivo reflectance confocal microscopy. Dermatol Surg 2010; 36:1099-110.

8 Hongcharu W, Dwyer P, Gonzalez S et al. Confirmation of onychomycosis by in vivo confocal microscopy. J Am Acad Dermatol 2000; 42:214-16.

9 Welzel J. [Optical coherence tomography]. Hautarzt 2010; 61:41620.

10 Welzel J, Lankenau E, Birngruber R et al. Optical coherence tomography of the human skin. J Am Acad Dermatol 1997; 37:958-63.

11 Mogensen M, Joergensen TM, Nurnberg BM et al. Assessment of optical coherence tomography imaging in the diagnosis of nonmelanoma skin cancer and benign lesions versus normal skin: observer-blinded evaluation by dermatologists and pathologists. Dermatol Surg 2009; 35:965-72.

12 Mogensen M, Thomsen JB, Skovgaard LT et al. Nail thickness measurements using optical coherence tomography and 20-MHz ultrasonography. Br J Dermatol 2007; 157:894-900.

13 Tsai TF, Maibach HI. How irritant is water? An overview. Contact Dermatitis 1999; 41:311-14.

14 Pinnagoda J, Tupker RA, Agner T et al. Guidelines for transepidermal water loss (TEWL) measurement. A report from the Standardization Group of the European Society of Contact Dermatitis. Contact Dermatitis 1990; 22:164-78.

15 Pinnagoda J, Tupker RA, Smit JA et al. The intra- and inter-individual variability and reliability of transepidermal water loss measurements. Contact Dermatitis 1989; 21:255-9.

16 Jemec GB, Agner T, Serup J. Transonychial water loss: relation to sex, age and nail-plate thickness. Br J Dermatol 1989; 121:443-6.

17 Kronauer C, Gfesser M, Ring J et al. Transonychial water loss in healthy and diseased nails. Acta Derm Venereol 2001; 81:175-7.

18 Murdan S, Hinsu D, Guimier M. A few aspects of transonychial water loss (TOWL): inter-individual, and intra-individual inter-finger, inter-hand and inter-day variabilities, and the influence of nail plate hydration, filing and varnish. Eur J Pharm Biopharm 2008; 70:684-9.

19 Egawa M, Ozaki Y, Takahashi M. In vivo measurement of water content of the fingernail and its seasonal change. Skin Res Technol 2006; 12:126-32. 